\title{
Oral perfluorooctane sulfonate (PFOS) lessens tumor development in the APC min mouse model of spontaneous familial adenomatous polyposis
}

Jeffrey Wimsatt ${ }^{1,2,5^{*}}$ (D, Meghan Villers ${ }^{1}$, Laurel Thomas', Stacey Kamarec ${ }^{1}$, Caitlin Montgomery ${ }^{1}$, Leo W. Y. Yeung ${ }^{3}$, Yanqing $\mathrm{Hu}^{4}$ and Kim Innes ${ }^{2}$

\begin{abstract}
Background: Colorectal cancer is the second most common cause of cancer deaths for both men and women, and the third most common cause of cancer in the U.S. Toxicity of current chemotherapeutic agents for colorectal cancer, and emergence of drug resistance underscore the need to develop new, potentially less toxic alternatives. Our recent cross-sectional study in a large Appalachian population, showed a strong, inverse, dose-response association of serum perfluorooctane sulfonate (PFOS) levels to prevalent colorectal cancer, suggesting PFOS may have therapeutic potential in the prevention and/or treatment of colorectal cancer. In these preliminary studies using a mouse model of familial colorectal cancer, the APC ${ }^{\text {min }}$ mouse, and exposures comparable to those reported in human populations, we assess the efficacy of PFOS for reducing tumor burden, and evaluate potential dose-response effects.

Methods: At 5-6 weeks of age, APC min mice were randomized to receive 0, 20, $250 \mathrm{mg}$ PFOS/ $/ \mathrm{kg}$ (females) or 0, 10, 50 and $200 \mathrm{mg} \mathrm{PFOS} / \mathrm{kg}$ (males) via their drinking water. At 15 weeks of age, gastrointestinal tumors were counted and scored and blood PFOS levels measured.

Results: PFOS exposure was associated with a significant, dose-response reduction in total tumor number in both male and female mice. This inverse dose-response effect of PFOS exposure was particularly pronounced for larger tumors ( $r^{2}$ for linear trend $=0.44$ for males, $p^{\prime} s<0.001$ ).

Conclusions: The current study in a mouse model of familial adenomatous polyposis offers the first experimental evidence that chronic exposure to PFOS in drinking water can reduce formation of gastrointestinal tumors, and that these reductions are both significant and dose-dependent. If confirmed in further studies, these promising findings could lead to new therapeutic strategies for familial colorectal cancer, and suggest that PFOS testing in both preventive and therapeutic models for human colorectal cancer is warranted.
\end{abstract}

Keywords: APC min mouse, Perfluorooctane sulfonate, PFOS, Colorectal cancer, Dose-response, Gender

\footnotetext{
* Correspondence: jefwimsatt@gmail.com

'Department of Medicine, School of Medicine, West Virginia University, Morgantown, WV 26506, USA

${ }^{2}$ Department of Epidemiology, School of Public Health, West Virginia

University, Morgantown, WV 26506, USA

Full list of author information is available at the end of the article
} 


\section{Background}

Colorectal cancer (colorectal cancer) is the second most common cause of cancer deaths in both men and women, and the third most common cause of cancer in the US [1]. Toxicity of current chemotherapeutic agents for colorectal cancer, and ongoing challenges with drug resistance suggest that new drug approaches continue to have value [2, 3].

Perfluoroalkyls and polyfluoroalkyls have been manufactured for over five decades; their unique-oilrepellence and high surface activity make them excellent surface protectants and surfactants. Some of these compounds are potent peroxisome proliferatoractivated receptor (PPAR) ligands, and have demonstrated anti-inflammatory effects in vitro [4] and in animal studies [5]; these effects are thought to operate via both PPAR-dependent and independent pathways [6]. Perfluorooctane sulfonate, (PFOS, $\mathrm{C}_{8} \mathrm{HF}_{17} \mathrm{O}_{3} \mathrm{~S}$ ), a well-studied perfluoro-surfactant, is a widespread environmental contaminant, has been detected in the plasma of virtually every human population worldwide [7-9]. PFOS is extremely stable in the environment, readily accumulates in people and animals, and has toxic properties; as a result several countries have voluntarily joined the Stockholm Convention to stop its use [10]. Lifetime exposure studies in rodents suggest PFOS can cause liver adenomas, whereas the evidence for cancer induction in humans remains equivocal, perhaps in part because most exposures levels are so low [11]. Hence, if shown of benefit, particularly at low doses, PFOS could suggest a novel mechanism for treating colorectal cancer.

Recent research suggests that PFOS may also have value as a chemopreventive and/or chemotherapeutic agent for colorectal cancer. In a cross-sectional study in a large Ohio Valley cohort (the C8 Health Project), we investigated the potential link between prevalent colorectal cancer and serum PFOS [12]. PFOS levels in this population were similar to those reported in the general U.S. population [12, 13], were comparable to or lower than those reported from non-occupational settings in other countries (e.g. $\leq 30 \mathrm{ng} / \mathrm{ml}$ ) $[14,15]$, and were well below levels reported in fluorochemical workers [16]. We found a strong, inverse, dose-response association between serum levels of PFOS and prevalent colorectal cancer that remained robust after adjustment for multiple possible confounders and persisted even at very low exposure levels [12]. However, while these findings suggest that PFOS may be protective against colorectal cancer, the cross-sectional nature of the data preclude determination of causality.

Here the potential chemotherapeutic value of PFOS is tested in $\mathrm{APC}^{\mathrm{min}}$ mice, a genetic model for familial adenomatous polyposis) in humans $[15,16]$.

\section{Methods}

\section{Chemicals and reagents}

For animal studies, potassium salt of PFOS (Sigma \#77282; heptadecafluorooctanesulfonic acid potassium salt) was purchased and dissolved in Millipore ${ }^{\circ}$ water containing 0.5\% Tween 20 (Sigma \#P2287). Bottles were made fresh weekly by addition from a stock solution. For analytical purposes, perfluorohexanoate, perfluoroheptanoate, perfluorooctanoate, potassium salts of perfluorohexanesulfonate, perfluorooctanesulfonate, and ${ }^{13} \mathrm{C}_{4}$ PFOS were obtained from Wellington Laboratories (Guelph, Ontario, Canada). The purity of all standards was over $98 \%$. Tetrabutylammonium hydrogen sulfate (99\%), ammonium acetate (>99\%), and ammonia $\left(\mathrm{NH}_{3}\right.$, $30 \%)$ were obtained from Sigma-Aldrich. LCMS grade methanol and methyl-tert-butyl ether (MTBE, > 99\%) were acquired from EMD Chemicals Inc. (Mississauga, ON). Oasis ${ }^{\circ}$ weak anion exchange (WAX; $6 \mathrm{~cm}^{3}, 150 \mathrm{mg}$, $30 \mu \mathrm{m}$ ) solid phase extraction (SPE) cartridges were purchased from Waters (Milford, MA).

All studies were approved by the Institutional Animal Care and Use Committee at West Virginia University. Animals were housed individually in standard ventilated barrier caging and fed standard mouse chow, and maintained on a $12: 12(\mathrm{~L}: \mathrm{D})$ hour light cycle. In two separate studies, female and male $\mathrm{APC}^{\text {min }}$ mice $(\mathrm{C} 57 \mathrm{BL} / 6 \mathrm{~J}$ $\left.\mathrm{Apc}^{\mathrm{Min}} / \mathrm{J}\right)$ were acquired from JAX at 6 and 5 weeks respectively, acclimated for 1 week, and randomized by treatment group. Animals in each group received Tween-20 vehicle or PFOS dissolved in Tween-20 in their drinking water. In an initial pilot study, female mice ( $n=8 /$ group) were exposed to $0.5 \%$ Tween-20 vehicle or Tween-20 with 20 , or $250 \mathrm{mg} / \mathrm{kg}$ PFOS target doses in their drinking water from 7-15 weeks of age based on estimated daily water consumption. Similarly, in the second study, male mice were exposed to vehicle or PFOS target doses of 10,50 and $200 \mathrm{mg} / \mathrm{kg}$ (all groups, $n=6$ ) provided at $6-15$ weeks of age. Animals were weighed twice weekly throughout the study period.

In both studies, animals were humanely euthanized with $\mathrm{CO}_{2}$, and the complete gastrointestinal tract from the stomach to the rectum was opened lengthwise and tumors were counted and categorized using direct visualization under 3 times magnification. Tumors were recognized by their characteristic gross morphology and categorized by location (small intestine, large intestine, cecum), size (using the average surface dimensions, they were scored as $<1 \mathrm{~mm}$ or $\geq 1 \mathrm{~mm}$ ), and if bleeding or not.

\section{Blood sampling}

In the male study, at 15 weeks of age, cardiac blood samples were collected under inhalant anesthesia into EDTA powdered tubes and the plasma collected and 
stored at $-80{ }^{\circ} \mathrm{C}$ until assayed. Plasma samples were shipped on ice to the University of Toronto, Department of Chemistry for PFOS measurement.

\section{PFOS assay \\ Sample extractions}

Mouse plasma samples were extracted using an ion-pair extraction method [17, 18]. Before extraction, mouse plasma samples were diluted with Milli-Q water (i.e., 10fold for control group and 1000 to 10000 -fold for treatment groups). In brief, in a $15 \mathrm{~mL}$ polypropylene tube, $1 \mathrm{~mL}$ of TBAS solution (adjusted to $\mathrm{pH} 10$ using 30\% aqueous $\mathrm{NH}_{3}$ ) was added to $1 \mathrm{~mL}$ of the diluted plasma sample; after the mixture was vortex-mixed for $30 \mathrm{~s}$, $5 \mathrm{~mL}$ of MTBE was added and was shaken on a horizontal shaker at 250 RPM for $20 \mathrm{~min}$; then the organic and aqueous layers were separated by centrifugation at 6000 RPM for $10 \mathrm{~min}$. The organic layer was decanted to a new tube. The sample was then extracted with another $5 \mathrm{~mL}$ aliquot of MTBE and the entire extraction procedure repeated. The MTBE aliquots were combined, evaporated to dryness under a gentle stream of nitrogen, and reconstituted in $1 \mathrm{~mL}$ of methanol for analysis.

Drinking water stock solution with $0.5 \%$ Tween-20 was extracted using a SPE-WAX cartridge [19]. The cartridge was first conditioned by passing a series of $4 \mathrm{~mL}$ of $0.1 \%$ $\mathrm{NH}_{4} \mathrm{OH}$ in methanol, $4 \mathrm{~mL}$ of methanol, and $4 \mathrm{~mL}$ of Milli-Q water; after that, $0.5 \mathrm{~mL}$ of the mouse drinking water stock was loaded onto the cartridge. After loading the sample, the cartridge was washed with $4 \mathrm{~mL}$ of $25 \mathrm{mM}$ ammonium acetate and dried under vacuum. Target fraction was eluted with $4 \mathrm{~mL} 0.1 \% \mathrm{NH}_{4} \mathrm{OH}$ in methanol and evaporated to dryness, and then reconstituted in $0.1 \mathrm{~mL}$ of methanol for analysis.

\section{Instrumental analysis}

Apart from PFOS, a suite of target Per- and polyfluorinated alkyl substances, C6-C8 perfluorinated carboxylic acids and perfluorohexane sulfonate were analyzed using an Acquity UPLC (Waters Corporation) and a API $4000 \mathrm{MS} / \mathrm{MS}$ (Applied Biosystem/MDS Sciex); an atmospheric electrospray interface operated in negative ionization mode was used. Chromatographic separation was performed on a Kinetex XB-C18 column $(50 \times$ $4.6 \mathrm{~mm}, 2.6$ um 100A), the column temperature was kept at $40{ }^{\circ} \mathrm{C}$, and $10 \mathrm{mM}$ ammonium acetate in both Milli-Q water and methanol were the mobile phases.

An internal calibration method using mass-labelled standard was used to quantify PFOS. The calibration curve was constructed with standard concentrations ranging from $0.5,1.0,2.5,5.0,10.0,20.0,50.0$, and $100.0 \mathrm{ng} /$ $\mathrm{mL}$. Standard deviations at each data point were $<20 \%$, with an $r^{2}>0.99$ for the calibration curve. The PFOS standard used in the present study was the linear isomer, while samples contained both branched and linear isomers; the concentrations reported for the present study included both linear and branched isomers, and were estimated based on the linear isomer standard. The limit of quantification $(0.5 \mathrm{ng} / \mathrm{mL})$ was evaluated based on the lowest concentration of standard on the calibration curve that could be accurately measured within $\pm 20 \%$ of its theoretical value and a signal-to-noise ratio $\geq 10$.

\section{Quality assurance and quality control}

Three procedural blanks (Milli-Q water) were run for every twelve sera samples to check for possible interference. Matrix recoveries $(n=3)$ using control mouse sera were performed prior to real sample analysis to ensure the reliability of the method. All target chemicals were spiked (10 ng) into the control samples, and the samples were extracted and analyzed following the same procedures as described above; matrix recoveries ranged 87$117 \%$ (supporting information, SI). Samples were analyzed in duplicate and the variability of the analysis was less than $10 \%$ as evaluated using ${ }^{13} \mathrm{C}_{4}$-PFOS recoveries, which likewise ranged from $88-110 \%$. In response to coeluting interferences at PFOS transition 499>80, the $499>99$ transition was used for quantification. Recoveries (spiked level: $0.5 \mathrm{ng}$ ) for water samples ranged 90 $107 \%$ (SI) using the same method. A quality control standard $(10 \mathrm{ng} / \mathrm{mL})$ was injected every ten samples to evaluate intensity change of the MS; samples were reanalyzed if the intensity of the standard varied $\pm 20 \%$ or more compared to those of the previous one.

\section{Statistical analyses}

Animals from the 2 studies represented different total doses, exposure periods and genders, so each study was analyzed separately. Both datasets were normality tested to assure parametric testing was appropriate. Initial ANOVA analysis was performed looking for treatment effects. Based on these results, summary statistics were calculated (means and standard errors), and assuming unequal variances, pairwise comparisons of treatment groups were made using two-sample $\mathrm{T}$ tests corrected using Tukey's criterion for multiple comparisons. Cecum masses were not included in analyses due to low tumor numbers. Slopes of mean body weights through time from the males exposed to vehicle or $200 \mathrm{mg} / \mathrm{kg}$ PFOS from 12-15 weeks were tested to determine if body weight trajectories are significantly different between the two groups.

\section{Results}

Of the original 24 female APC $^{\text {min }}$ mice, one from the $20 \mathrm{mg} / \mathrm{kg}$ dose group developed breast cancer, and 4 from the $250 \mathrm{mg} / \mathrm{kg}$ dose group lost $>10 \%$ body weight, so they were lost to follow-up before 15 weeks. Findings 
reported for this pilot study, illustrated in Fig. 1a, are based on the remaining animals. Initially, ANOVA analysis using treatment as the independent variable and total tumor number as the dependent variable, revealed $p$-values of 0.010 and 0.019 for females and males respectively. At 15 weeks in females, total tumor numbers in both treatment groups averaged significantly lower than those in the control group ( $p=0.02$ and 0.009 for $20 \mathrm{mg} / \mathrm{kg}$ and $250 \mathrm{mg} / \mathrm{kg}$, respectively). Likewise, animals in the $250 \mathrm{mg} / \mathrm{kg}$ group averaged significantly fewer tumors than in the $20 \mathrm{mg} / \mathrm{kg}$ group $(p=0.04)$. Collectively, these findings indicate increasing tumor reductions with rising PFOS dose.

Findings of the study in male mice are shown in Fig. 1b, and suggest a similar dose-response relationship overall.

Again, total tumor load in all treatment groups averaged lower than that in the control group at 15 weeks, with the largest effect in the highest dose group ( $p$ 's $=0.051,0.068$, and 0.003 for vehicle vs. 10,50 , and $200 \mathrm{mg} / \mathrm{kg}$, respectively). Likewise, while response in the lowest PFOS dose group did not differ significantly from that in the moderate dose group $(p>0.1)$, total tumor count in animals receiving PFOS doses of $200 \mathrm{mg} / \mathrm{kg}$ was significantly reduced relative to both the $10 \mathrm{mg} / \mathrm{kg}(p=0.006)$ and $50 \mathrm{mg} / \mathrm{kg}$ dose groups $(p=0.02)$, again suggesting greater tumor reduction at higher PFOS doses.

As indicated in Fig. 1c, the inverse, dose-response effect of PFOS exposure was particularly pronounced for tumors $\geq 1 \mathrm{~mm}$ in size. For males, PFOS showed a strong inverse linear association to large tumor numbers $\left(r^{2}=0.44\right)$ with a $p<0.001$. For tumors $<1 \mathrm{~mm}$, this association did not hold $(p=0.76)$. In both studies, bleeding tumors were rare, appeared to be evenly distributed across dose groups, and their occurrence appeared unrelated to either tumor size or location.

Figure 2 shows the effect of PFOS dose on body weight in males. Weight gain decreased with dose in the final weeks of the study, with the between group differences increasingly prominent after 11 weeks. As expected, weight gain reductions were particularly pronounced in the $200 \mathrm{mg} / \mathrm{kg}$ group, with the slope of body weight change in this group becoming negative by 12 weeks, with body weight trending lower than for the vehicle controls from $12-15$ weeks $(p=0.06)$. Weight loss in this high dose group late in the study likely reflects a developing toxic effect of PFOS, and is consistent with the weight loss observed at higher doses in the female study. As illustrated in Fig. 3, plasma PFOS levels at 15 weeks of age indicate that PFOS accumulation increased with dose, including estimated levels of both linear and branched isoforms.

\section{Discussion}

In these two preliminary studies of male and female APC $^{\text {min }}$ mice, total tumor number decreased significantly with increasing PFOS dose, with the highest dose groups showing the largest effects. The observed doseresponse relationship was particularly evident in larger tumors, suggesting a possible inhibitory effect of PFOS on tumor formation. Since PFOS administration in our mouse model was initiated prior to tumor development,

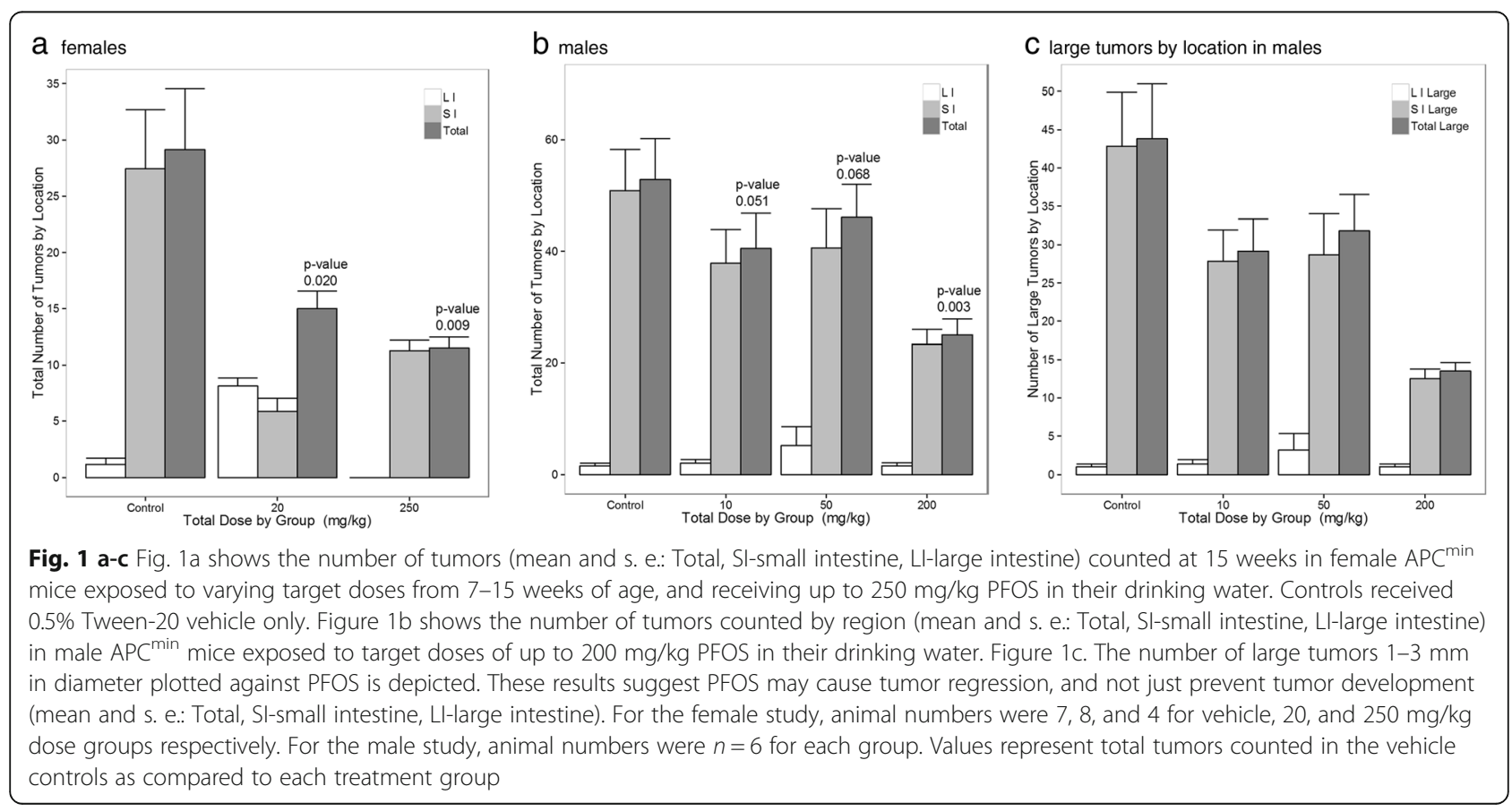




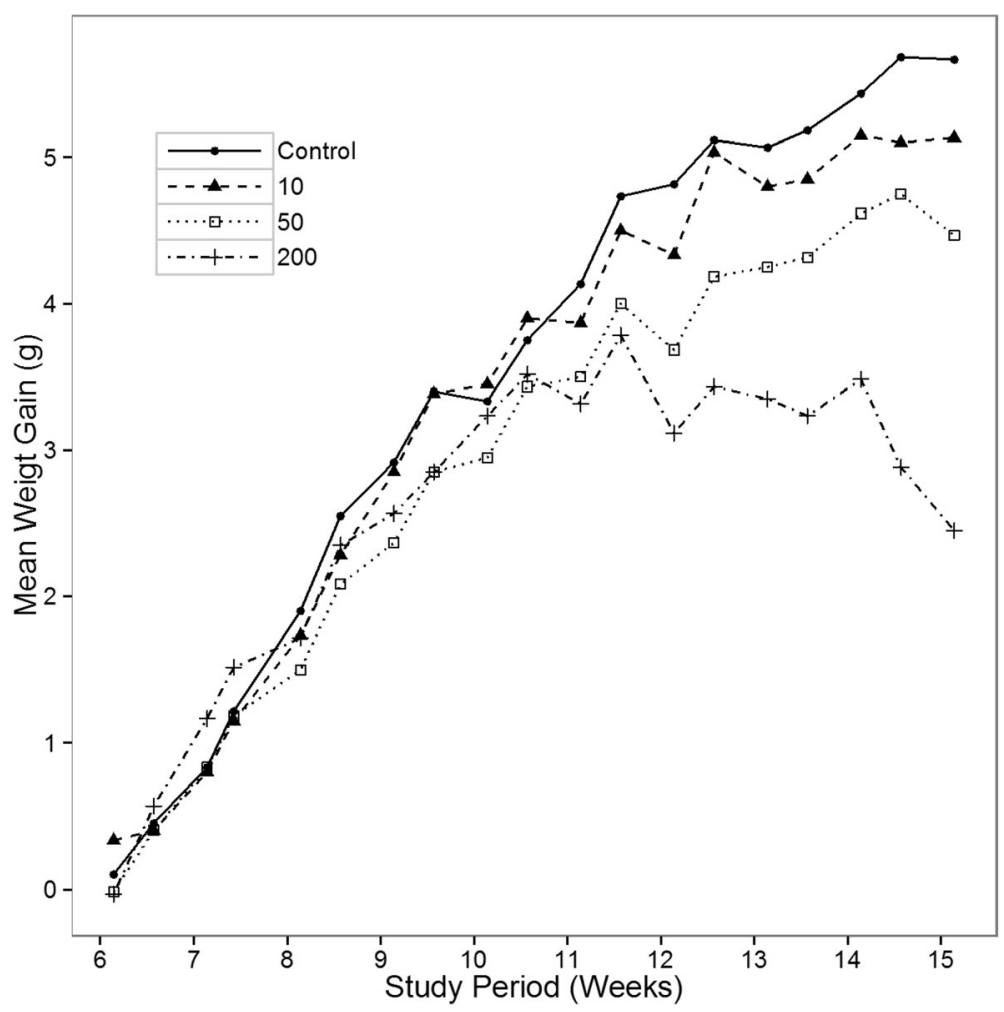

Fig. 2 Shown are plasma PFOS levels (mean and s. e.) from male APC ${ }^{m i n}$ mice at 15 weeks of age by dose administered. Total linear and branched PFOS levels are shown. As expected, levels increased with PFOS dose

the observed reduction in tumor burden may reflect effects on both tumor initiation and tumor progression. Notably, the number of $1-3 \mathrm{~mm}$ tumors decreased significantly with increasing PFOS dose (Fig. 1c), suggesting that PFOS may not only inhibit development, but may halt progression and even possibly induce tumor regression. If PFOS induces tumor regression, reduction of larger tumors may lead to a corresponding increase in the number of tumors $<1 \mathrm{~mm}$ diameter, thus potentially attenuating the observed effect of PFOS on total tumor number and helping to explain the stronger effects observed for larger tumors. Collectively, these findings suggest that PFOS has a significant, dose-dependent inhibitory effect on gastrointestinal tumor formation in this established genetic mouse model of familial adenomatous polyposis.

Results of these preliminary experimental studies are broadly consistent with findings from our recent epidemiological investigation in a large population of Appalachian adults exposed to PFOA-contaminated drinking water. In this cross-sectional study, serum PFOS levels showed a strong, inverse dose-response association with prevalent colorectal cancer that remained robust after adjustment for multiple potential confounders [12]. However, while findings of this epidemiological investigation likewise suggest a possible protective effect of PFOS on colorectal cancer, the cross-sectional nature of the data limit causal inference. Although implications for nonfamilial colorectal cancer remain unclear, the current study in a mouse model of familial adenomatous polyposis offers the first experimental evidence that chronic exposure to PFOS in drinking water can reduce formation of gastrointestinal tumors, and that these reductions are both significant and dose-dependent.

In our present animal study, we provided PFOS in the drinking water to simulate chronic human PFOS exposure $[20,21]$. Liver enzymes can be induced by PFOS exposure in mice [22], and toxicity indicated by weight loss [23] was observed here. In other studies, higher PFOS doses have been administered over shorter periods by oral bolus $[5,24]$ without evident toxic effects; however, our data suggest toxicity likely develops at a lower overall dose when PFOS is delivered slowly over time [5, 24]. Here progressive weight loss was observed at doses of $200 \mathrm{mg} / \mathrm{kg}$ or higher, indicating this dose is near the maximum tolerated dose for this mouse strain and delivery method over this time frame. Fortunately, measurement of plasma PFOS levels in male mice at 15 weeks indicated that drinking water administration at all doses resulted in plasma levels substantially higher than those associated colorectal cancer reduction in humans [12]. In common, PFOS appeared beneficial in human colorectal cancer and in $\mathrm{APC}^{\mathrm{min}}$ mice. However, the 


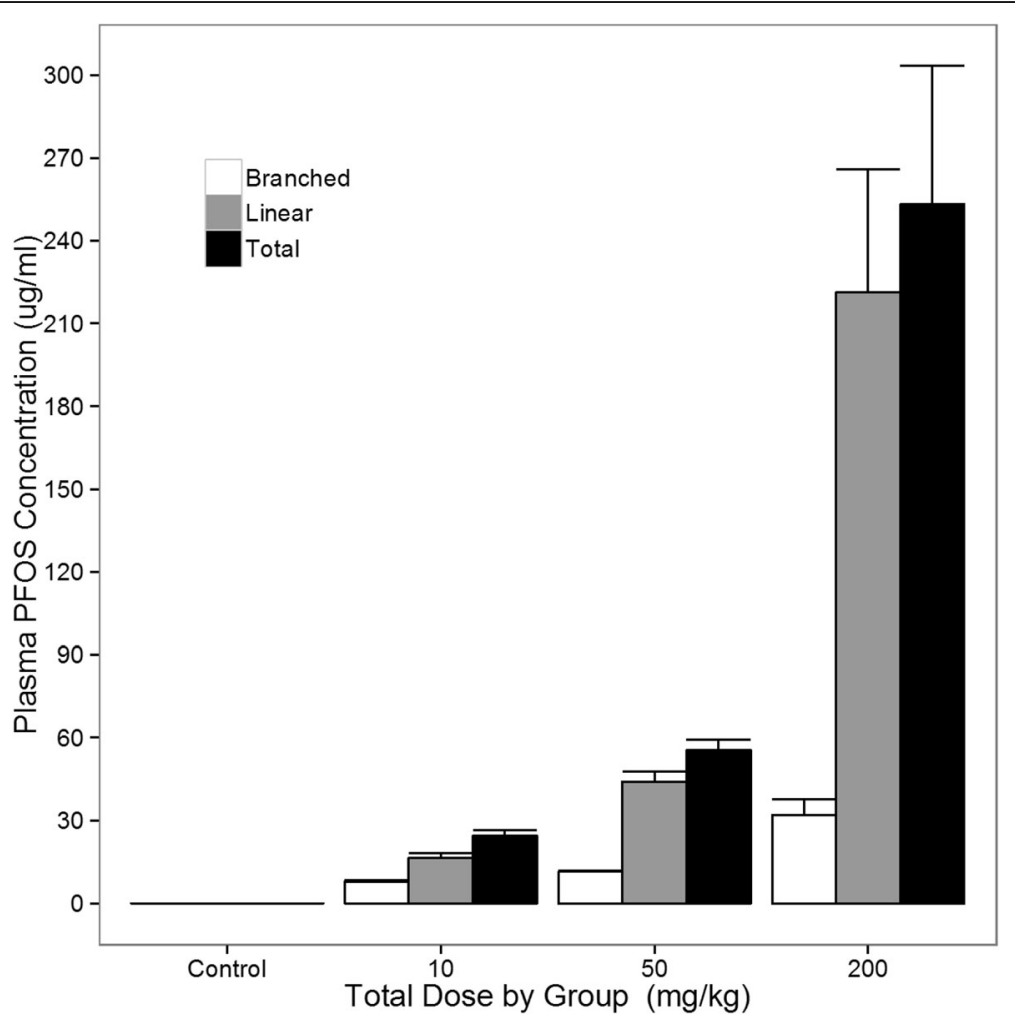

Fig. 3 Shown is the average body weight (mean and s. e.) by dose group from 6-15 weeks of age weeks in male mice. As can be seen, body weight appeared to slow in proportion to PFOS dose. At approximately 12 weeks of age, the $200 \mathrm{mg} / \mathrm{kg}$ group stopped exhibiting weight increases altogether. Table 1 depicts the gender, dose groups, animal numbers and duration of exposure for the animals completing each study

former effect was observed in humans which metabolize PFOS differently from rodents, and where the effect was substantially based on the acquired non-familial form of colorectal cancer. In the mouse model, PFOS undergoes a greater degree of metabolism, has a shorter half-life, and counters a genetic predisposition to colorectal cancer. Further studies should investigate the adverse consequences of this agent under therapeutic conditions; even so, this study provides a possible direction to pursue in regard to familial colorectal cancer.

Although PFOS is widely distributed in the environment $[25,26]$ and has been detected in human populations worldwide [9, 27-30], non-occupational blood levels in humans are well below those reported toxic in lab animals [31-33]. The half-life of PFOS is reported to be $<40$ days in mice [34], and contrasts dramatically with the estimated 4-5 year half-life documented in humans [35]. It appears that PFOS in rodents is handled in a manner similar to fatty acids, and consequently induces hormonal, peroxisomal and P450 enzyme gene activation [36]. The increased PFOS half-life in humans compared to rodents may be in part due to PFOS inhibition of human cytochrome activity [37]; cytochrome activity inhibition could also reduce the influence of toxic metabolites which may explain higher degrees of toxicity as commonly reported in rodents. In addition, PFOS renal reabsorption and recycling have also been shown to contribute to a long half-life in humans and monkeys [38]. Cancer risk with prolonged chronic exposure was suggested at high doses in rats [11], although consistent evidence for elevated tumor risk with PFOS exposure in humans is lacking [11].

Each PFAS has a unique biological and toxicological profile that limits extrapolation across compounds or

Table 1 Depicts the gender, dose groups, animal numbers and duration of exposure for the animals completing each study

\begin{tabular}{|c|c|c|c|c|}
\hline Trial & $\begin{array}{l}\text { Dose Groups in mg/kg } \\
(\mathrm{N})\end{array}$ & Age at First Exposure & Treatment Duration & Age at Tumor Counts \\
\hline Females & $0(8), 20\left(7^{\mathrm{a}}\right), 250\left(4^{\mathrm{b}}\right)$ & 7 weeks & 8 weeks & 15 weeks \\
\hline Males & $0(6), 10(6), 50(6), 200(6)$ & 6 weeks & 9 weeks & 15 weeks \\
\hline
\end{tabular}

${ }^{a}$ One animal developed breast cancer and was dropped from study; ${ }^{\text {b}}$ four animals had > 10\% weight loss before 15 weeks and were lost to follow-up. "0" dose animals received vehicle only 
model species [11]. Potential mechanisms of PFOS action relevant to its effect on tumor development and progression are still ill-defined, but are not surprising given the large number of genes (e.g. 400 in rats) PFOS appears to influence [36]. Possibilities include antiinflammatory effects via prostanoid pathways, PPAR receptor mediated actions, immune effects, or other as yet unrecognized mechanisms. PFOS may serve an antiinflammatory role via its influence on downstream transcriptional regulators such as NF-kB [6]. Phospholipase A2 is inhibited by PFOS in rats; this could, in turn, block the production of arachidonic acid as a substrate for prostaglandindin $\mathrm{H}$ synthase elaboration of prostanoids [36]. Similarly, $\mathrm{PGE}_{2}$ has been shown to be a potent inducer of adenoma formation in $\mathrm{APC}^{\mathrm{min}}$ mice [39], and tumor growth [40] was similarly increased by an agonist, where both were mediated through the PPAR $\delta$ receptor. Adenoma formation by $\mathrm{PGE}_{2}$ was removed in mice missing this receptor [41]. PFOS also significantly stimulates both PPAR $\alpha$ and PPAR $\gamma$ [42], which could also modulate tumor growth $[43,44]$. PFOS serves as a partial agonist and induces PPAR $\alpha$ mediated effects at high doses [45], other effects via other PPAR receptor isotypes [46], and produces significant immunomodulatory effects in mice [47]. In PPAR $\alpha$ KO mice, PPAR $\alpha$ independent nuclear receptor mediated pathways and downstream effects were noted [6], including suppression of T-cell dependent antibody production, and modulation of immune cell and cytokine synthesis (e.g. TNF $\alpha$ and IL-6) [6]. While PFOS appears to stimulate mouse and human PPAR receptors [42, 46] when screened in cell lines, robust in vivo evidence for direct PPAR receptor mediation is lacking, and human PPAR $\alpha$ expression is considerably reduced compared to in rodents; if so, this may thus lessen the importance of this pathway in human familial adenomatous polyposis or acquired colorectal cancer [6]. Even so, PPAR $\alpha$ stimulation in human colorectal cancer lines is moderately pro-inflammatory and stimulates prostaglandin $\mathrm{H}$ synthase- 2 expression $[48,49]$. The potential impact of PFOS directly on the Wnt- $\beta$-catenin signal transduction pathway also warrants closer examination [50]. The "min" defect causes catenin retention and ultimately the Wnt gene group to become canonically activated; gastrointestinal polyp formation is one direct consequence [51]. The putative role of PFOS in blocking this process would be a plausible mechanism to explain its efficacy in this model system. Alternative pathways could also be affected [52]. Other influences of PFOS, by interacting with dietary constituents [53, 54], or steroidogenic enzyme and hormone disruptive effects cannot be ruled in or out [55]. Here, both mouse genders benefited from PFOS exposure, arguing against a differential role as pertains to specific sex hormones.
In recent human cross-sectional studies, chronic PFOS exposure has been associated with modest, adverse changes in serum lipid profiles [56-58]. Similarly, elevated blood levels of PFOS have been associated with increased likelihood of early onset menopause [59] and altered thyroid function [59]. However, in contrast to findings from animal studies, including our study in $\mathrm{APC}^{\text {min }}$ mice, significant adverse effects of PFOS exposure have been rarely been documented in humans, even in pregnant women and highly exposed fluorochemical plant workers $[16,58$, $60,61]$. Even with its complex disposition in humans, PFOS appears well tolerated at environmental levels (e.g. Danish National Birth Cohort Study [62]: PFOS mean $35.3 \mathrm{ng} / \mathrm{ml}$; range $6.4-106.7 \mathrm{ng} / \mathrm{ml}$; elsewhere $0-30 \mathrm{ng} / \mathrm{ml}$ [15, 63-65],) levels also associated with colorectal cancer protection [66]. Occupational exposures in chemical factory workers are up to 40 times higher, yet adverse outcomes at these levels, even among at risk pregnant women, are rare $[16,60]$. At high occupational exposure levels, an association between PFOS and bladder cancer was reported [65]; however, this association was questioned more recently [16, 58, 61]. Therefore, although PFOS has been decried as an environmental contaminant, it might still have therapeutic value at low levels. If shown to be effective for colorectal cancer prevention and/or treatment in humans, PFOS may offer an option that is significantly safer, lower cost, and less toxic than alternative therapies [67].

One potential limitation relates to reverse causality, i.e., the possibility that tumor formation may reduce PFOS absorption, and thus, blood PFOS levels. However, this is unlikely given the massive surface area of the gastrointestinal tract and the inherent lipid solubility of PFOS. Moreover, serum PFOS generally correlates well with liver concentrations [61], suggesting that serum is a reasonably good systemic indicator of PFOS exposure in humans [68]. Similarly in a mouse model of familial adenomatous polyposis, blood levels corresponded to target dosing levels and predicted tumor reduction, again indicating that PFOS absorption reflects oral exposure. Another theoretical argument is that a parent compound is broken down to make PFOS, and it may be this compound, rather than PFOS itself that led to the reduction in colorectal cancer observed in our previous epidemiological study [12]. However, PFOS exposure had a clear benefit here.

Strengths of this study include identifying a beneficial role for PFOS as a chemo-preventive or therapy in a familial model of colorectal cancer, irrespective of gender. In addition, the development of a slow delivery method and estimates of an effective working dose range by this delivery method were determined. Finally, toxic effects were easily identified using simple body weight monitoring. Limitations include the absence of more dose 
groups to better define the dose-response relationship, with the eventual goal of developing a reliable PFOS therapeutic profile.

While effective against this animal model of familial adenomatous polyposis, PFOS efficacy in the more common acquired form of colorectal cancer is another important focus for future investigations. Planned studies will seek to determine a mechanism, such as might be derived from isolated cell cultures, an optimal dose in vivo using xenograft models. A mechanistic understanding of PFOS action, from the impetus provided here, may lead to successful new colorectal cancer treatment approaches.

\section{Conclusions}

Using $\mathrm{APC}^{\mathrm{min}}$ mice of both genders, we show that perfluorooctane sulfonate (PFOS) reduces gastrointestinal tumor burden in this well-established general model of human colorectal cancer, and also a specific model for familial adenomatous polyposis in a dose-related manner. Our results represent a proof of concept based on our previously published epidemiological study that found a protective dose-response relationship between PFOS levels and reduced likelihood of colorectal cancer from a human population.

\section{Abbreviations \\ ANOVA: Analysis of variance; C57BL/6 J-Apc Min/J mice: APC min mice; LCMS: Liquid chromatography mass spectrometry; MTBE: Methyl-tert-butyl ether; PFOA: Perfluorooctanoic acid; PFOS, $\mathrm{C}_{8} \mathrm{HF}_{17} \mathrm{O}_{3} \mathrm{~S}$ : Perfluorooctane sulfonate; PPAR: Peroxisome proliferator-activated receptor; SPE: Solid phase extraction; WAX: Oasis ${ }^{\oplus}$ weak anion exchange;}

\section{Acknowledgements}

The Authors would like to thank A. Forrisi and Dr. V. Rajendran for technical assistance and Dr. Patrick Callery for reading and commenting on the analytical methods.

\section{Funding}

$\mathrm{NIH}$ grant to JW (U54GM104942).

\section{Authors' contributions \\ JW provided the conceptual framework, paper writing, and sought and provided funding support. MV, LT, KK, and CM collected data, and managed the project day to day. LY-performed assay validations and assayed samples. YH provided power analyses and statistical expertise. KI provided critical background leading to the discovery and it relevance to human cancer. All authors have read and approved the manuscript as written.}

\section{Competing interests}

Coi certifications are on file with WVU. The authors declare that they have no competing interests.

\section{Consent for publication}

All authors have consented to publish the present manuscript s presented here.

\section{Ethics approval}

IACUC approval from West Virginia University was secured to cover this animal work.

\section{Primary data}

Cannot be posted publicly due to proprietary limitations and patent infringement-"Not Applicable".

\section{Author details \\ 'Department of Medicine, School of Medicine, West Virginia University, Morgantown, WV 26506, USA. ${ }^{2}$ Department of Epidemiology, School of Public Health, West Virginia University, Morgantown, WV 26506, USA. ${ }^{3}$ Man-Technology-Environment (MTM) Research Centre, School of Science and Technology, Örebro University, Fakultetsgatan 1, Örebro SE-70182, Sweden. ${ }^{4}$ Department of Statistics, West Virginia University, Morgantown, WV 26506, USA. ${ }^{5}$ West Virginia University, 186 HSCN, 1 Medical Center Drive, Morgantown, WV 26508, USA.}

Received: 17 February 2016 Accepted: 11 October 2016

Published online: 08 December 2016

\section{References}

1. Colorectal (Colon) Cancer Statistics [http://www.cdc.gov/cancer/colorectal/ statistics/index.htm]. Accessed 2 Nov 2016.

2. Sartore-Bianchi A, Bencardino K, Cassingena A, Venturini F, Funaioli C, Cipani T, Amatu A, Pietrogiovanna L, Schiavo R, Di Nicolantonio F, et al. Therapeutic implications of resistance to molecular therapies in metastatic colorectal cancer. Cancer Treat Rev. 2010;36 Suppl 3:S1-5.

3. Arber N, Spicak J, Racz I, Zavoral M, Breazna A, Gerletti P, Lechuga MJ, Collins N, Rosenstein RB, Eagle CJ, et al. Five-year analysis of the prevention of colorectal sporadic adenomatous polyps trial. Am J Gastroenterol. 2011; 106(6):1135-46.

4. Corsini E, Sangiovanni E, Avogadro A, Galbiati V, Viviani B, Marinovich M, Galli CL, Dell'Agli M, Germolec DR. In vitro characterization of the immunotoxic potential of several perfluorinated compounds (PFCs). Toxicol Appl Pharmacol. 2012;258(2):248-55.

5. Mollenhauer MA, Bradshaw SG, Fair PA, McGuinn WD, Peden-Adams MM. Effects of perfluorooctane sulfonate (PFOS) exposure on markers of inflammation in female B6C3F1 mice. J Environ Sci Health A Tox Hazard Subst Environ Eng. 2011;46(2):97-108

6. DeWitt JC, Shnyra A, Badr MZ, Loveless SE, Hoban D, Frame SR, Cunard R, Anderson SE, Meade BJ, Peden-Adams MM, et al. Immunotoxicity of perfluorooctanoic acid and perfluorooctane sulfonate and the role of peroxisome proliferator-activated receptor alpha. Crit Rev Toxicol. 2009; 39(1):76-94.

7. Calafat AM, Wong L-Y, Kuklenyik Z, Reidy JA, Needham LL. Polyfluoroalkyl Chemicals in the U.S. Population: Data from the National Health and Nutrition Examination Survey (NHANES) 2003-2004 and Comparisons with NHANES 1999-2000. Environ Health Perspect. 2007;115(11):1596-602.

8. Hanssen L, Rollin H, Odland JO, Moe MK, Sandanger TM. Perfluorinated compounds in maternal serum and cord blood from selected areas of South Africa: results of a pilot study. J Environ Monit. 2010;12(6):1355-61.

9. Chunli $C$, Yonglong $L$, Xiang $Z$, Jing G, Tieyu W, Yajuan S, Wenyou $H$, Jing $L$. A review of spatial and temporal assessment of PFOS and PFOA contamination in China. Chem Ecol. 2009;25(3):163-77.

10. Stockholm Convention on Persistent Organic Pollutants (POPs)[PFOS, its salts, and perfluorooctane sulfonyl fluoride (PFOSF) -restrictions on production and use] [http://www.oecd.org/ehs/pfc/]. Accessed 2 Nov 2016.

11. Kennedy G, Symons M. Chapter 12. Carcinogencity of perfluoroalkyl compounds. In: Dewitt J, ed. Toxicological effects of perfluoroalkyl and polyfluoroalkyl substances, molecular and integrative toxicology. Basel: Springer; 2015. p. 265-304.

12. Innes KE, Wimsatt JH, Frisbee S, Ducatman AM. Inverse association of colorectal cancer prevalence to serum levels of perfluorooctane sulfonate (PFOS) and perfluorooctanoate (PFOA) in a large Appalachian population. BMC Cancer. 2014;14:45

13. Calafat AM, Kuklenyik Z, Reidy JA, Caudill SP, Tully JS, Needham LL. Serum concentrations of 11 polyfluoroalkyl compounds in the u.s. population: data from the national health and nutrition examination survey (NHANES). Environ Sci Technol. 2007;41(7):2237-42

14. Kannan K, Corsolini S, Falandysz J, Fillmann G, Kumar KS, Loganathan BG, Mohd MA, Olivero J, Van Wouwe N, Yang JH, et al. Perfluorooctanesulfonate and related fluorochemicals in human blood from several countries. Environ Sci Technol. 2004;38(17):4489-95. 
15. Yeung LW, Robinson SJ, Koschorreck J, Mabury SA. Part II. A temporal study of PFOS and its precursors in human plasma from two German cities in 1982-2009. Environ Sci Technol. 2013;47(8):3875-82.

16. Alexander $\mathrm{BH}$, Olsen GW. Bladder cancer in perfluorooctanesulfonyl fluoride manufacturing workers. Ann Epidemiol. 2007;17(6):471-8.

17. D'Eon JC, Crozier PW, Furdui VI, Reiner EJ, Libelo EL, Mabury SA. Observation of a commercial fluorinated material, the polyfluoroalkyl phosphoric acid diesters, in human sera, wastewater treatment plant sludge, and paper fibers. Environ Sci Technol. 2009;43(12):4589-94.

18. Hansen KJ, Clemen LA, Ellefson ME, Johnson HO. Compound-specific, quantitative characterization of organic fluorochemicals in biological matrices. Environ Sci Technol. 2001;35(4):766-70.

19. Taniyasu S, Kannan K, So MK, Gulkowska A, Sinclair E, Okazawa T, Yamashita N. Analysis of fluorotelomer alcohols, fluorotelomer acids, and short- and long-chain perfluorinated acids in water and biota. J Chromatogr A. 2005;1093(1-2):89-97.

20. Hu XC, Andrews DQ, Lindstrom AB, Bruton TA, Schaider LA, Grandjean P, Lohmann R, Carignan CC, Blum A, Balan SA, Higgins CP, Sunderland EM. Detection of Poly- and Perfluoroalkyl Substances (PFASs) in U.S. Drinking Water Linked to Industrial Sites, Military Fire Training Areas, and Wastewater Treatment Plants. Environ Sci Technol Lett. 2016 Oct 11;3(10):344-350. Epub 2016 Aug 9

21. Knox SS, Jackson T, Javins B, Frisbee SJ, Shankar A, Ducatman AM. Implications of early menopause in women exposed to perfluorocarbons. J Clin Endocrinol Metab. 2011;96(6):1747-53.

22. Tan F, Jin Y, Liu W, Quan X, Chen J, Liang Z. Global liver proteome analysis using iTRAQ labeling quantitative proteomic technology to reveal biomarkers in mice exposed to perfluorooctane sulfonate (PFOS). Environ Sci Technol. 2012;46(21):12170-7.

23. Qazi MR, Nelson BD, DePierre JW, Abedi-Valugerdi M. High-dose dietary exposure of mice to perfluorooctanoate or perfluorooctane sulfonate exerts toxic effects on myeloid and B-lymphoid cells in the bone marrow and these effects are partially dependent on reduced food consumption. Food Chem Toxicol. 2012;50(9):2955-63.

24. Dong GH, Zhang $Y H$, Zheng $L$, Liang ZF, Jin YH, He QC. Subchronic effects of perfluorooctanesulfonate exposure on inflammation in adult male C57BL/6 mice. Environ Toxicol. 2012;27:285-96.

25. Giesy JP, Kannan K. Global distribution of perfluorooctane sulfonate in wildlife. Environ Sci Technol. 2001:35(7):1339-42.

26. Kunacheva C, Fujii S, Tanaka S, Seneviratne ST, Lien NP, Nozoe M, Kimura K, Shivakoti BR, Harada H. Worldwide surveys of perfluorooctane sulfonate (PFOS) and perfluorooctanoic acid (PFOA) in water environment in recent years. Water Sci Technol. 2012;66(12):2764-71.

27. Eom J, Choi J, Kim J, Kim Y. A survey of exposure level and lifestyle factors for perfluorooctanoate and perfluorooctane sulfonate in human plasma from selected residents in Korea. Int J Environ Res Public Health. 2014;11(7):7231-41.

28. Eriksen KT, Sorensen M, McLaughlin JK, Tjonneland A, Overvad K, RaaschouNielsen O. Determinants of plasma PFOA and PFOS levels among 652 Danish men. Environ Sci Technol. 2011;45(19):8137-43.

29. Harada KH, Yang HR, Moon CS, Hung NN, Hitomi T, Inoue K, Niisoe T, Watanabe T, Kamiyama S, Takenaka K, et al. Levels of perfluorooctane sulfonate and perfluorooctanoic acid in female serum samples from Japan in 2008, Korea in 1994-2008 and Vietnam in 2007-2008. Chemosphere. 2010:79(3):314-9.

30. Lindstrom AB, Strynar MJ, Libelo EL. Polyfluorinated compounds: past, present, and future. Environ Sci Technol. 2011:45(19):7954-61.

31. Grasty RC, Bjork JA, Wallace KB, Wolf DC, Lau CS, Rogers JM. Effects of prenatal perfluorooctane sulfonate (PFOS) exposure on lung maturation in the perinatal rat. Birth Defects Res B Dev Reprod Toxicol. 2005;74(5):405-16.

32. Luebker DJ, Case MT, York RG, Moore JA, Hansen KJ, Butenhoff JL. Twogeneration reproduction and cross-foster studies of perfluorooctanesulfonate (PFOS) in rats. Toxicology. 2005;215(1-2):126-48.

33. DeWitt JC, Peden-Adams MM, Keller JM, Germolec DR. Immunotoxicity of perfluorinated compounds: recent developments. Toxicol Pathol. 2012:40(2):300-11.

34. Chang SC, Noker PE, Gorman GS, Gibson SJ, Hart JA, Ehresman DJ, Butenhoff JL. Comparative pharmacokinetics of perfluorooctanesulfonate (PFOS) in rats, mice, and monkeys. Reprod Toxicol. 2012;33(4):428-40.

35. Olsen GW, Burris JM, Ehresman DJ, Froehlich JW, Seacat AM, Butenhoff JL, Zobel LR. Half-life serum elimination of perfluorooctanesulfonate, perfluorohexanesulfonate, and perfluorooctanoate in retired fluorochemical production workers. Environ Health Perspect. 2007;115(9):1298-305.
36. Hu W, Jones PD, Celius T, Giesy JP. Identification of genes responsive to PFOS using gene expression profiling. Environ Toxicol Pharmacol. 2005; 19(1):57-70

37. Narimatsu S, Nakanishi R, Hanioka N, Saito K, Kataoka H. Characterization of inhibitory effects of perfluorooctane sulfonate on human hepatic cytochrome P450 isoenzymes: focusing on CYP2A6. Chem Biol Interact. 2011;194(2-3):120-6.

38. Andersen ME, Clewell 3rd HJ, Tan YM, Butenhoff JL, Olsen GW. Pharmacokinetic modeling of saturable, renal resorption of perfluoroalkylacids in monkeys-probing the determinants of long plasma half-lives. Toxicology. 2006;227(1-2):156-64

39. Hansen-Petrik MB, McEntee MF, Jull B, Shi H, Zemel MB, Whelan J. Prostaglandin E(2) protects intestinal tumors from nonsteroidal anti-inflammatory druginduced regression in Apc(Min/+) mice. Cancer Res. 2002;62(2):403-8.

40. Gupta RA, Wang D, Katkuri S, Wang H, Dey SK, DuBois RN. Activation of nuclear hormone receptor peroxisome proliferator-activated receptor-delta accelerates intestinal adenoma growth. Nat Med. 2004;10(3):245-7.

41. Wang D, Wang H, Shi Q, Katkuri S, Walhi W, Desvergne B, Das SK, Dey SK, DuBois RN. Prostaglandin E(2) promotes colorectal adenoma growth via transactivation of the nuclear peroxisome proliferator-activated receptor delta. Cancer Cell. 2004:6(3):285-95.

42. Vanden Heuvel J, Thompson J, Frame S, Gillies P. Differential activation of nuclear receptors by perfluorinated fatty acid analogs and natural fatty acids: a comparison of human, mouse, and rat peroxisome proliferatoractivated receptor-alpha, -beta, and -gamma, liver $X$ receptor-beta, and retinoid X receptor-alpha. Toxicol Sci. 2006;92(2):476-89.

43. Niho N, Takahashi M, Kitamura T, Shoji Y, Itoh M, Noda T, Sugimura T, Wakabayashi K. Concomitant suppression of hyperlipidemia and intestinal polyp formation in Apc-deficient mice by peroxisome proliferator-activated receptor ligands. Cancer Res. 2003:63(18):6090-5.

44. Shipley JM, Hurst CH, Tanaka SS, DeRoos FL, Butenhoff JL, Seacat AM, Waxman DJ. trans-activation of PPARalpha and induction of PPARalpha target genes by perfluorooctane-based chemicals. Toxicol Sci. 2004;80(1):151-60.

45. Ye L, Zhao B, Yuan K, Chu Y, Li C, Zhao C, Lian QQ, Ge RS. Gene expression profiling in fetal rat lung during gestational perfluorooctane sulfonate exposure. Toxicol Lett. 2012;209(3):270-6.

46. Takacs ML, Abbott BD. Activation of mouse and human peroxisome proliferatoractivated receptors (alpha, beta/delta, gamma) by perfluorooctanoic acid and perfluorooctane sulfonate. Toxicol Sci. 2007;95(1):108-17.

47. Fair PA, Driscoll E, Mollenhauer MAM, Bradshaw SG, Yun SH, Kannan K, Bossart GD, Keil DE, Peden-Adams MM. Effects of environmentally relevant levels of perfluorooctane sulfonate on clinical parameters and immunological functions in B6C3F1 mice. J Immunotoxicol. 2011;8(1):17-29. Epub 2011 Jan 24 doi:103109/1547691X2010527868 2011, 8(1):17-29.

48. Oshio H, Abe T, Onogawa T, Ohtsuka H, Sato T, li T, Fukase K, Muto M, Katayose $Y$, Oikawa M, et al. Peroxisome proliferator-activated receptor alpha activates cyclooxygenase-2 gene transcription through bile acid transport in human colorectal cancer cell lines. J Gastroenterol. 2008;43(7):538-49.

49. Liao Y, Wang J, Huang QS, Fang C, Kiyama R, Shen H, Dong S. Evaluation of cellular response to perfluorooctane sulfonate in human umbilical vein endothelial cells. Toxicol In Vitro. 2012:26(3):421-8.

50. Brembeck FH, Wiese M, Zatula N, Grigoryan T, Dai Y, Fritzmann J, Birchmeier W. BCL9-2 promotes early stages of intestinal tumor progression. Gastroenterology. 2011;141(4):1359-70.

51. Taketo MM. Wnt signaling and gastrointestinal tumorigenesis in mouse models. Oncogene. 2006;25(57):7522-30.

52. Cai J, Maitra A, Anders RA, Taketo MM, Pan D. beta-Catenin destruction complex-independent regulation of Hippo-YAP signaling by APC in intestinal tumorigenesis. Genes Dev. 2015;29(14):1493-506.

53. Paulsen JE, Alexander J. Growth stimulation of intestinal tumours in Apc(Min/+) mice by dietary L-methionine supplementation. Anticancer Res. 2001:21(5):3281-4

54. Song J, Medline A, Mason JB, Gallinger S, Kim YI. Effects of dietary folate on intestinal tumorigenesis in the apcMin mouse. Cancer Res. 2000;60(19):5434-40.

55. Du G, Hu J, Huang H, Qin Y, Han X, Wu D, Song L, Xia Y, Wang X. Perfluorooctane sulfonate (PFOS) affects hormone receptor activity, steroidogenesis, and expression of endocrine-related genes in vitro and in vivo. Environ Toxicol Chem. 2013;32(2):353-60.

56. Frisbee S, Shankar A, Knox S, Steenland K, Savitz D, Fletcher T, Ducatman A. Perfluorooctanoic Acid, perfluorooctanesulfonate, and serum lipids in 
children and adolescents: results from the $c 8$ health project. Arch Pediatr Adolesc Med. 2010;164(9):860-9.

57. Steenland K, Tinker S, Frisbee S, Ducatman A, Vaccarino V. Association of perfluorooctanoic acid and perfluorooctane sulfonate with serum lipids among adults living near a chemical plant. Am J Epidemiol. 2009; 170(10):1268-78.

58. Butenhoff JL, Olsen GW, Pfahles-Hutchens A. The applicability of biomonitoring data for perfluorooctanesulfonate to the environmental public health continuum. Environ Health Perspect. 2006;114(11):1776-82.

59. Knox SS, Jackson T, Frisbee SJ, Javins B, Ducatman AM. Perfluorocarbon exposure, gender and thyroid function in the $\mathrm{C} 8$ Health Project. J Toxicol Sci. 2011;36(4):403-10.

60. Grice MM, Alexander BH, Hoffbeck R, Kampa DM. Self-reported medical conditions in perfluorooctanesulfonyl fluoride manufacturing workers. J Occup Environ Med. 2007:49(7):722-9.

61. Alexander BH, Olsen GW, Burris JM, Mandel JH, Mandel JS. Mortality of employees of a perfluorooctanesulphonyl fluoride manufacturing facility. Occup Environ Med. 2003;60(10):722-9.

62. Chun Yuan F, McLaughlin JK, Tarone RE, Olsen J. Perfluorinated chemicals and fetal growth: a study within the Danish national birth cohort. Environ Health Perspect. 2007;115(11):1677-82.

63. Vassiliadou I, Costopoulou D, Ferderigou A, Leondiadis L. Levels of perfluorooctanesulfonate (PFOS) and perfluorooctanoate (PFOA) in blood samples from different groups of adults living in Greece. Chemosphere. 2010;80(10):1199-206.

64. Jin Y, Saito N, Harada KH, Inoue K, Koizumi A. Historical trends in human serum levels of perfluorooctanoate and perfluorooctane sulfonate in Shenyang, China. Tohoku J Exp Med. 2007;212(1):63-70.

65. Nakayama S, Harada K, Inoue K, Sasaki K, Seery B, Saito N, Koizumi A. Distributions of perfluorooctanoic acid (PFOA) and perfluorooctane sulfonate (PFOS) in Japan and their toxicities. Environ Sci. 2005;12(6):293-313.

66. Appleman TD, Higgins CP, Quinones O, Vanderford BJ, Kolstad C, ZeiglerHolady JC, Dickenson ER. Treatment of poly-and perfluoroalkyl substances in U.S. full-scale water treatment systems. Water Res. 2014;51:246-55.

67. Numico G, Longo V, Courthod G, Silvestris N. Cancer survivorship: long-term side-effects of anticancer treatments of gastrointestinal cancer. Curr Opin Oncol. 2015:27(4):351-7.

68. Karrman A, Domingo JL, Llebaria X, Nadal M, Bigas E, van Bavel B, Lindstrom G. Biomonitoring perfluorinated compounds in Catalonia, Spain: concentrations and trends in human liver and milk samples. Environ Sci Pollut Res Int. 2009;17(3):750-8

\section{Submit your next manuscript to BioMed Central and we will help you at every step:}

- We accept pre-submission inquiries

- Our selector tool helps you to find the most relevant journal

- We provide round the clock customer support

- Convenient online submission

- Thorough peer review

- Inclusion in PubMed and all major indexing services

- Maximum visibility for your research

Submit your manuscript at wuw biomedcentral.com/submit

) Biomed Central 